\title{
Breaking the therapeutic ceiling in drug development in ulcerative colitis
}

Dahham Alsoud, MD'; Bram Verstockt, MD, PhD ${ }^{1,2}$; Claudio Fiocchi, $\mathrm{MD}^{3}$ and Séverine Vermeire, $\mathrm{MD}$, $\mathrm{PhD}^{1,2}$

1. KU Leuven, Department of Chronic Diseases, Metabolism and Ageing, Translational Research in Gastrointestinal disorders (TARGID) - IBD Unit, Leuven, BELGIUM

2. University Hospitals Leuven, Department of Gastroenterology and Hepatology, KU Leuven, Leuven, BELGIUM

3. Department of Inflammation and Immunity, Lerner Research Institute, and Department of Gastroenterology, Hepatology and Nutrition, Digestive Disease and Surgery Institute, Cleveland Clinic, Cleveland, OH, USA.

Short title: Drug development in UC

\section{Corresponding author}

Séverine Vermeire, MD, PhD

Department of Gastroenterology and Hepatology, University Hospitals Leuven

Herestraat 493000 Leuven, Belgium.

Phone: $0032(0) 16344218$

E-mail severine.vermeire@uzleuven.be

@ibdleuven@AlsoudDahham @bverstockt @SeverineVermei1

Total word count: 2633

Total number of figures / tables: 3 / 0

Total number of references: 62 


\begin{abstract}
:
Advances in the intricate pathogenesis of ulcerative colitis (UC) have triggered a broad endeavour in drug development over the last two decades, resulting in the advent of several biological agents and small molecules. Although the increase in therapeutic options is a positive event, remission rates by new therapeutic agents in induction trials remain at a very modest $20-30 \%$, and seemingly facing a socalled therapeutic ceiling. This situation requires a critical appraisal and highlights the need for alternative drug development strategies. We here objectively itemise the boundaries of therapeutic efficacy in UC, provide possible explanations for the shortcomings, and propose solutions to achieve better therapeutic outcomes in UC.
\end{abstract}


INTRODUCTION: the current therapeutic landscape in UC:

Ulcerative colitis (UC) is a chronic idiopathic inflammatory condition affecting the mucosal and submucosal layers of the colon. ${ }^{1}$ The disease is characterized by a relapsing-remitting nature and, when insufficiently controlled, leads to progressive organ damage with impaired function, anatomical changes (lead-pipe colon), risk of dysplasia and/or cancer.

Before 2000, therapeutic options for UC were limited to corticosteroids, 5-aminosalicylates, thiopurines, and calcineurin inhibitors. This changed around the new millennium with the introduction of biological therapies targeting tumor necrosis factor (TNF) and later other molecules targeting different biological pathways. ${ }^{2}$

Merely 20 years after the approval by the European Medicines Agency (EMA) and the United States Food and Drug Administration (FDA) of infliximab for UC, no less than three classes of biologicals (antitumor necrosis factor alpha (anti-TNF alpha), anti-integrins, inhibitors of interleukins (ILs) 12/23) and the first small molecules (Janus kinase (JAK) inhibitors) have become available to treat moderate-tosevere UC. In addition, new agents from these and other classes, such as sphingosine 1 phosphate receptor 1 (S1PR1) modulators, will presumably soon enter the therapeutic armamentarium with positive data from phase 3 clinical trials. ${ }^{3}$

This recent revolution in UC therapeutics has led to significant positive changes in disease outcomes, as evidenced by the decreasing colectomy, mortality and colorectal cancer rates. ${ }^{4-6}$ However, physicians find themselves nowadays flooded with therapeutic options and have difficulties in deciding which therapy to start in which patient at what time.

\section{The therapeutic ceiling in UC: contributing factors, challenges and solutions}

Despite the current wealth in treatment options, therapeutic success rates are still far from optimal. In fact, it is striking that remission rates - defined by the Mayo score as a combination of clinical improvement in stool frequency and blood loss with improvement on endoscopy - seem not to surpass an upper limit of $20-30 \%$ in induction trials (Figure 1). ${ }^{7-16}$ What may be responsible for this ceiling and how could we break it?

\section{Inclusion of refractory patients in clinical trials}

Similar to Crohn's disease (CD), UC is a progressive disease in a significant proportion of patients with increasing refractoriness with longstanding disease and repeated therapeutic exposures. ${ }^{17}$ Hence, missing an early potential "therapeutic window of opportunity" may lower success rates of future alternative therapies. This could partially explain the lower efficacy rates in bio-failure patients in comparison to bio-naïve patients, as consistently observed with all compounds introduced after the introduction of anti-TNFs. Given that more and more patients recruited to new phase 2 or 3 trials have been previously exposed to multiple biologics and small molecules, demonstrating efficacy with novel agents becomes even more challenging. Therefore, selecting less refractory patients, with shorter disease duration and limited therapeutic exposure, may result in better efficacy, although this tradeoff is intrinsically associated with slower recruitment rates. It was recently shown that although the number of active randomized controlled trials (RCTs) for IBD more than doubled between 2011 and 2018 , the RCT participation rates decreased from $1.1 \%$ to $0.7 \%{ }^{18}$

Some patients are refractory to many (or even all) available agents, despite a short disease duration, and will likely have lower response rates when recruited in clinical trials. Based on this observation, it seems reasonable to search for molecular biomarkers that could identify patients with multirefractory 
disease. These patients may benefit more from early surgical intervention or novel therapies that target their specific disease regulatory mechanisms using a multi-omic approach, as explained later.

\section{Positioning of available drugs}

The increasing number of new agents puts pressure on both the health care system and on payers. Many regulatory authorities will therefore impose cheaper anti-TNF biologicals or biosimilars as the first line of treatment, and physicians would only be allowed to start newer therapies with other mechanisms of action (MOA) after the initial therapeutic approach fails. Presently, scarce evidence is available on which biologic should be used and under which circumstances. ${ }^{19}$ Singh et al. provided a ranking based on network meta-analyses of data from clinical trials. ${ }^{20}$ There are inherent limitations to such analysis, as those registry trials have never been designed as head-to-head or comparative effectiveness systems addressing relative drug performance. That said, head-to-head trials - like VARSITY and HIBISCUS - would undoubtedly help in positioning the available agents, together with competitive price settings for the newer small molecules. ${ }^{21}$ However, without incorporating predictive biomarkers, results emerging from head-to-head trials will only be informative on a population level and will not aid therapeutic decisions for the individual patients.

\section{Personalized assignment of licensed therapies}

As mentioned earlier, molecular drivers of UC pathogenesis almost certainly differ between patients, even though no direct evidence exists at the moment which, in turn, would lead to non-responsiveness to a drug targeting of a specific molecule not pathogenically involved. This would introduce a selection bias into head-to-head trials and hamper an ideal positioning of available therapies in treatment algorithms. Hence, biomarkers predicting responsiveness or non-responsiveness to both available and new drugs should be incorporated first in clinical trials and later in clinical practice to guide informed treatment decisions. Several molecular markers predicting response to anti-TNF alpha and other agents have been proposed, but they still need independent validation before wide implementation, ${ }^{22-}$ ${ }^{26}$ and their accuracy is yet unclear.

\section{Undefined therapeutic classes combination}

Given the complexity of immune responses and the multiple abnormalities associated with UC, it seems reasonable to assume that combining drugs with different mechanisms of actions may achieve better remission rates. Several case series and case reports demonstrated that dual biological therapy (DBT) is effective in inducing or restoring remission after monotherapies failures. ${ }^{27-32}$ Results from ongoing clinical trials (NCT02764762, investigating the combination of vedolizumab, adalimumab and methotrexate in CD; NCT03662542, investigating the combination of guselkumab and golimumab in UC) are eagerly awaited to address legitimate safety concerns, raise hope for improved efficacy rates and define the correct place in therapeutic algorithms.

\section{Variable endoscopic disease assessment at baseline}

Baseline endoscopic severity has been shown to influence the chance of achieving endoscopic remission in $C D .{ }^{33}$ Therefore, ensuring that endoscopic severity is equally assessed in all trial arms is pivotal in obtaining objective information about the real efficacy of investigated agents. In this respect, the popular Mayo score suffers from several weaknesses, and unbiased artificial intelligence (AI)-based systems, like machine learning, may prove to be more accurate in defining disease severity than the human eye, as explained later.

\section{Confounders in outcome assessment}

Placebo response rates tend to be high in UC and have been brought down to some degree with central 
reading of the endoscopic findings, but substantial interobserver variability still persists and compromises the accuracy of outcome assessment. ${ }^{34}$ The development of operator-independent objective and reliable automated systems to read endoscopic, ultrasound and histologic images is a field of great interest. Several operator-independent, machine learning-based endoscopic scoring systems have been introduced and correlated well with available endoscopic scales. ${ }^{35-38}$ As the performance of these objective scoring systems is strictly dependent on training sets, they are built on, it is vital that these sets include images that represent a challenge for endoscopists in real life situations, like images with suboptimal quality and those with low disease activity. Reproducibility of the developed systems has yet to be validated in prospective well-designed studies.

\section{The need for new scoring systems}

In order to obtain accurate data on the efficacy of investigational drugs, scoring systems should consider all aspects of endoscopic inflammation, not only the severity but also the extent. The Mayo endoscopic subscore (MES), the most commonly used one, does not take into account the extent of inflammation and may therefore not accurately reflect the effect a drug may have in healing the mucosa. Even when a substantial decrease in the inflammatory appearance is seen, the score may provide a misleading disease activity unless all affected segments are equally healed. A modified, simple version of the Mayo endoscopic subscore (MMES) taking into account the number of inflamed segments has been developed and correlated very well with clinical, biological and histological disease activity. ${ }^{39}$ Its clinical value is yet to be validated in long-term multicentric trials.

\section{Confounders in concomitant baseline treatments}

In most conditions, including UC, high or relatively high placebo-response rates are common, lowering the chances to identify a significant benefit from new therapies even with a reasonable sample size. One possible contributing factor to the high placebo response rates is that some response can also be achieved by low but chronic use of corticosteroids, 5 -aminosalicylates or immunomodulators whose use is still allowed in clinical trials. ${ }^{40}$ The most recent phase 3 trials with ustekinumab, tofacitinib, filgotinib, etrolizumab or ozanimod included $40-50 \%$ of patients on corticosteroids during induction. The required and often long (8-12 weeks) wash-out period after failing a particular therapy is increasing steroid use as patients with active disease are in need of some treatment before they can be screened and randomized for trials investigating new therapies. Besides, spontaneous remission due to the natural history of UC has also been reported without the use of treatments. ${ }^{41}$

\section{Timing of "treat to clear", an ambitious step further!}

Endpoints to assess response to therapy have evolved from the introduction of steroid-free remission, to endoscopic healing and, most recently, histologic healing. The latter has been introduced since histological inflammation may persist in seemingly healed mucosa by routine endoscopic examination. ${ }^{42}$ As persistent histologic inflammation is associated with higher relapse rates, a new "treat to clear" approach - beyond "treat to target" ${ }^{43}$ - that combines symptomatic, endoscopic and histological remission has recently been proposed. ${ }^{44}$ Advocating for this approach is supported by emerging data associating amelioration of histological inflammation, full restoration of the architecture and absence of inflammatory infiltrate with favourable long-term outcomes, more than endoscopic remission alone. ${ }^{45,46}$ Furthermore, Christensen et al. demonstrated that full resolution of histological inflammation in all segments of the colon might be needed to minimize the risk of flares. ${ }^{45}$ In its position paper on UC histopathology, the European Crohn's and Colitis Organisation (ECCO) acknowledges that histologic remission may predicts lower rates of UC complications; however, more evidence needs to be generated before adopting this new target in clinical practice. ${ }^{47}$ In their metaanalysis, Magro et al. failed to show superiority of histological endpoints over clinical and endoscopic 
remission in distinguishing the efficacy of investigational therapeutics from that of placebo in clinical trials. ${ }^{48}$ However, in the pooled analysis the trials assessed histologic response early at the end of induction phase, and one can question whether such an ambitious goal would require longer time to be achieved. Therefore, future trials will need to define the optimal timing of histological assessment, its cost-benefits and potential superiority as new endpoints over the current endoscopic and clinical endpoints. The VERDICT trial (NCT04259138) is exactly addressing these research questions and results of this trial may help reshape treatment strategies.

Finally, to ensure validity and reproducibility of histologic assessments, standardization of biopsy sampling procedures and reaching a consensus over the ideal histologic reporting indices are essential steps. ${ }^{49-51}$ ECCO recommended the use of the Robarts histopathology index or the Nancy index for randomized clinical trials, and defined histologic remission as the absence of intraepithelial neutrophils, erosion and ulceration. ${ }^{47}$

\section{The lack of new and flexible trials design}

The multi-target approach typically applied in UC, that combines clinical, biological, endoscopic and more recently histological outcomes, along with the inclusion of "all comers" with various phenotypes (age at onset, disease extent, extra-intestinal manifestation of disease, previous exposure to therapies...), use of concomitant medications (steroids, immunomodulators...) and presumably various molecular profiles, has led to trials with traditional rigid design that consumes huge resources and requires long time periods. ${ }^{52}$ In this context, a more flexible and less sophisticated "adaptive" study design would allow to reduce the needed number of recruited patients, increase cost savings, shorten of trials' durations, and allocate patients to the best treatment arm. Here, predetermined modifications on trial design are performed upon an interim analysis of data collected at pre-scheduled time-points. Adaptive design can be of different types, each offering a solution for a problem of currently used design. In their study to compare the efficacy of cangrelor and clopidogrel after percutaneous coronary intervention ( $\mathrm{PCl}$ ), Bhatt et al. were able to anticipate a possible loss of statistical power due to insufficient sample size using an "adaptive sample size re-estimation design". ${ }^{53}$ An "adaptive dose-finding design" helped Labrèze et al. in determining the propranolol dose with the highest efficacy in patients with infantile haemangiomas, which allowed the investigators to offer enrolled patients the drug dosage with the highest efficacy, reduce study duration and save costs. ${ }^{54}$ Furthermore, "biomarker-derived adaptive trials", which would be of a tremendous help in head-tohead trials in UC, has already helped in evaluating therapeutics of breast cancer where patients were randomized to different regimens based on their response rate assessed using a 10 biomarker signatures. ${ }^{55}$ However, great attention and efforts should be paid to the initial planning phase of such new designs with strict interim-analyses follow-up and subsequent adjustments in order to avoid results misinterpretation and guarantee the statistical integrity of the study. ${ }^{56,57}$ Therefore, experts should devise clear recommendations on how a new design would help a better drug development in UC. $^{58}$

\section{"Out of the box" approach in therapeutic targets discovery}

Current evidence acknowledges that UC has a complex aetiopathogenesis and the naïve assumption that this disease can be controlled by targeting only few specific pathways of the immune system should be abandoned. ${ }^{59}$ Instead, multiple different pathways are likely to be activated in any given UC patient, and these pathways may change during disease evolution due to environmental exposures (diet, smoking, pollutants, etc.), metagenomic, epigenetic, proteomic and metabolomic modifications, structural tissue abnormalities, and so on. These changes mutually influence and modify each other in highly complex ways that cannot be detected by current clinical estimates and require integration of 
the underlying molecular events that can only be achieved using unbiased artificial intelligence (Al)based analytical technics. ${ }^{60}$ Using bioinformatic computational algorithms, multi-omic integrative approaches will allow a categorisation of UC patients into subgroups based on shared molecular patterns (IBD interactomes) irrespective of their traditional clinical classifications (rectal UC, left-sided UC, pancolitis). This approach offers several clear advantages: first, it objectively proves the heterogeneity of UC pathobiology and its degree; second, it can identify molecular biomarkers that separate UC patients into distinct homogeneous subgroups; third, it explains why only some patients respond to any particular medication; fourth, it avoids giving medications targeting molecules not involved in the disease process; fifth, it leads to the identification of essential hubs (central regulators) that underlie the pathogenesis of each UC subgroup; sixth, once the hubs are identified, they become the specific targets for new drugs developed based on proven implication in disease causation or maintenance (Figure 2). ${ }^{61}$ This "out of the box" approach represents the essence of a precision medicine approach, which will break the current therapeutic ceiling in UC to become the standard way to evaluate and treat patients in the next decade. ${ }^{62}$

\section{CONCLUSION:}

Recent efforts in expanding the therapeutic UC armamentarium can only be applauded. However, as remission rates are still far from optimal, introducing innovative ideas in basic, translational and clinical aspects of drug development is essential in the relentless search to achieve breakthroughs in UC therapeutics that address the needs of patients, physicians and health regulators (Figure 3 ). To address and eliminate the various above-mentioned challenges efforts should simultaneously be devoted to three major areas: first, move to adaptive trial designs for new agents targeting the appropriate patients depending on the mode of action of that agent, using robust objective scoring systems for clinical, endoscopic and histological outcomes; second, define the relative efficacy of existing drugs using head-to-head trials in order to formulate clear treatments algorithms at the population level and incorporate predictive markers to tailor these drugs to the individual patient; third, start using stateof-the-art analytical (Al-based bioinformatics) techniques to classify patients in molecularly defined biomarker-positive clinical subgroups that can be treated with highly specific drugs targeting the molecular hubs controlling the underlying disease network.

\section{REFERENCES:}

1 Ulcerative colitis. Nature Reviews Disease Primers 2020; 6: 73.

2 Harbord M, Eliakim R, Bettenworth D, et al. Third European evidence-based consensus on diagnosis and management of ulcerative colitis. Part 2: Current management. Journal of Crohn's and Colitis 2017; 11: 769-84.

3 Sabino J, Verstockt B, Vermeire S, Ferrante M. New biologics and small molecules in inflammatory bowel disease: an update. Therapeutic Advances in Gastroenterology. 2019; 12.

https://doi.org/10.1177/1756284819853208.

4 Burisch J, Katsanos KH, Christodoulou DK, et al. Natural Disease Course of Ulcerative Colitis During the First Five Years of Follow-up in a European Population-based Inception Cohort-An Epi-IBD Study. Journal of Crohn's and Colitis 2019; 13: 198-208. 
5 Alatab S, Sepanlou SG, Ikuta K, et al. The global, regional, and national burden of inflammatory bowel disease in 195 countries and territories, 1990-2017: a systematic analysis for the Global Burden of Disease Study 2017. The Lancet Gastroenterology and Hepatology 2020; 5: 17-30.

6 Castaño-Milla C, Chaparro M, Gisbert JP. Systematic review with meta-Analysis: The declining risk of colorectal cancer in ulcerative colitis. Alimentary Pharmacology and Therapeutics. 2014; 39: 64559.

7 Rutgeerts P, Sandborn WJ, Feagan BG, et al. Infliximab for Induction and Maintenance Therapy for Ulcerative Colitis. New England Journal of Medicine 2005; 353: 2462-76.

8 Sandborn WJ, van Assche G, Reinisch W, et al. Adalimumab induces and maintains clinical remission in patients with moderate-to-severe ulcerative colitis. Gastroenterology 2012; 142. https://doi.org/10.1053/j.gastro.2011.10.032.

9 Sandborn WJ, Feagan BG, Marano C, et al. Subcutaneous golimumab induces clinical response and remission in patients with moderate-to-severe ulcerative colitis. Gastroenterology 2014; 146: 85-95.

10 Feagan BG, Rutgeerts $P$, Sands BE, et al. Vedolizumab as induction and maintenance therapy for ulcerative colitis. New England Journal of Medicine 2013; 369: 699-710.

11 Dotan I, Panés J., Duvall A. et al. Etrolizumab compared with adalimumab or placebo as induction therapy for ulcerative colitis: results from the randomized, phase 3 HIBISCUS I \& II trials. UEG Week; Amsterdam; 10-13 October 2020.

12 Peyrin-Biroulet L., Hart A.L., Bossuyt P. et al. Etrolizumab as induction and maintenance therapy in patients with ulcerative colitis previously exposed to anti-tumor necrosis factor agent: the randomized, phase 3 HICKORY trial. UEG Week; Amsterdam; 10-13 October 2020.

13 Sands BE, Sandborn WJ, Panaccione R, et al. Ustekinumab as Induction and Maintenance Therapy for Ulcerative Colitis. New England Journal of Medicine 2019; 381: 1201-14.

14 Sandborn WJ, Su C, Sands BE, et al. Tofacitinib as Induction and Maintenance Therapy for Ulcerative Colitis. New England Journal of Medicine 2017; 376: 1723-36.

15 Feagan B.G., Loftus, Jr. E.V. et al. Efficacy and safety of filgotinib as induction therapy for patients with moderately to severely active ulcerative colitis: results from the phase $2 \mathrm{~b} / 3$ SELECTION study. UEG Week; Amsterdam; 10-13 October 2020.

16 Sandborn W., D'Haens G.R., Wolf D.C. et al. Ozanimod as induction therapy in moderate-tosevere ulcerative colitis: results from the phase 3, randomized, double-blind, placebo-controlled TRUE NORTH study. UEG Week; Amsterdam; 10-13 October 2020.

17 Torres J, Billioud V, Sachar DB, Peyrin-Biroulet L, Colombel JF. Ulcerative colitis as a progressive disease: The forgotten evidence. Inflammatory Bowel Diseases. 2012; 18: 1356-63.

18 Johnson C, Barnes EL, Zhang X, Long MD. Trends and Characteristics of Clinical Trials Participation for Inflammatory Bowel Disease in the United States: A Report From IBD Partners. Crohn's \& Colitis 360 2020; 2. https://doi.org/10.1093/crocol/otaa023

19 Raine T, Verstockt B, de Cruz P. Immune therapies in ulcerative colitis: are we beyond anti-TNF yet? The Lancet Gastroenterology and Hepatology. 2020; 5: 794-6. 
20 Singh S, Murad MH, Fumery M, Dulai PS, Sandborn WJ. First- and Second-Line Pharmacotherapies for Patients With Moderate to Severely Active Ulcerative Colitis: An Updated Network Meta-Analysis. Clinical Gastroenterology and Hepatology. 2020; 18: 2179-2191.e6.

21 Pouillon L, Travis S, Bossuyt P, Danese S, Peyrin-Biroulet L. Head-to-head trials in inflammatory bowel disease: past, present and future. Nature Reviews Gastroenterology and Hepatology 2020; 17: 365-76.

22 West NR, Hegazy AN, Owens BMJ, et al. Oncostatin M drives intestinal inflammation and predicts response to tumor necrosis factor-neutralizing therapy in patients with inflammatory bowel disease. Nature Medicine 2017; 23: 579-89.

23 Bertani L, Fornai $M$, Fornili $M$, et al. Serum oncostatin $M$ at baseline predicts mucosal healing in Crohn's disease patients treated with infliximab. Alimentary Pharmacology \& Therapeutics 2020; 52: 284-91.

24 Verstockt B, Verstockt S, Dehairs J, et al. Low TREM1 expression in whole blood predicts anti-TNF response in inflammatory bowel disease. EBioMedicine 2019; 40: 733-42.

25 Verstockt B, Verstockt S, Creyns B, et al. Mucosal IL13RA2 expression predicts nonresponse to anti-TNF therapy in Crohn's disease. Alimentary Pharmacology and Therapeutics 2019; 49: 572-81.

26 Noor NM, Verstockt B, Parkes M, Lee JC. Personalised medicine in Crohn's disease. The lancet. Gastroenterology \& hepatology. 2020; 5: 80-92.

27 Buer LCT, Høivik ML, Warren DJ, Medhus AW, Moum BA. Combining Anti-TNF- $\alpha$ and Vedolizumab in the Treatment of Inflammatory Bowel Disease: A Case Series. Inflammatory Bowel Diseases 2018; 24: 997-1004.

28 Kwapisz L, Raffals LE, Bruining DH, et al. Combination Biologic Therapy in Inflammatory Bowel Disease: Experience from a Tertiary Care Center. Clinical Gastroenterology and Hepatology 2020; 0. https://doi.org/10.1016/j.cgh.2020.02.017.

29 Glassner K, Oglat A, Duran A, et al. The use of combination biological or small molecule therapy in inflammatory bowel disease: A retrospective cohort study. Journal of Digestive Diseases 2020; 21: 264-71.

30 Yang E, Panaccione N, Whitmire N, et al. Efficacy and safety of simultaneous treatment with two biologic medications in refractory Crohn's disease. Alimentary Pharmacology \& Therapeutics 2020; 51: 1031-8.

31 Fumery M, Yzet C, Brazier F. Letter: combination of biologics in inflammatory bowel diseases. Alimentary Pharmacology \& Therapeutics 2020; 52: 566-7.

32 Privitera G, Onali S, Pugliese D, et al. Dual Targeted Therapy: A Possible Option for the Management of Refractory Inflammatory Bowel Disease. Journal of Crohn's and Colitis 2021; 15: 335-9.

33 Narula N, Wong ECL, Aruljothy A, et al. Ileal and Rectal Ulcer Size Affects the Ability to Achieve Endoscopic Remission: A Post hoc Analysis of the SONIC Trial. The American journal of gastroenterology 2020; 115: 1236-45. 
34 Feagan BG, Sandborn WJ, D'Haens G, et al. The role of centralized reading of endoscopy in a randomized controlled trial of mesalamine for ulcerative colitis. Gastroenterology 2013; 145: 149157.e2.

35 Bossuyt $\mathrm{P}$, Nakase $\mathrm{H}$, Vermeire $\mathrm{S}$, et al. Automatic, computer-aided determination of endoscopic and histological inflammation in patients with mild to moderate ulcerative colitis based on red density. Gut 2020; 69: 1778-86.

36 Takenaka K, Ohtsuka K, Fujii T, et al. Development and Validation of a Deep Neural Network for Accurate Evaluation of Endoscopic Images From Patients With Ulcerative Colitis. Gastroenterology 2020; 158: 2150-7.

37 Yao H, Najarian K, Gryak J, et al. Fully automated endoscopic disease activity assessment in ulcerative colitis. Gastrointestinal Endoscopy 2020; published online Aug 15.

https://doi.org/10.1016/j.gie.2020.08.011.

38 Bossuyt P, de Hertogh G, Eelbode T, Vermeire S, Bisschops R. Computer-Aided Diagnosis With Monochromatic Light Endoscopy for Scoring Histologic Remission in Ulcerative Colitis.

Gastroenterology 2020; 160: 23-5.

39 Lobatón T, Bessissow T, de Hertogh G, et al. The Modified Mayo Endoscopic Score (MMES): A New Index for the Assessment of Extension and Severity of Endoscopic Activity in Ulcerative Colitis Patients. Journal of Crohn's \& colitis 2015; 9: 846-52.

40 Harbord M, Eliakim R, Bettenworth D, et al. Third European evidence-based consensus on diagnosis and management of ulcerative colitis. Part 2: Current management. Journal of Crohn's and Colitis 2017; 11: 769-84.

41 Jairath V, Zou GY, Parker CE, et al. Placebo response and remission rates in randomised trials of induction and maintenance therapy for ulcerative colitis. Cochrane Database of Systematic Reviews. 2017; 2017. https://doi.org/10.1002/14651858.CD011572.pub2.

42 Bryant R v., Burger DC, Delo J, et al. Beyond endoscopic mucosal healing in UC: Histological remission better predicts corticosteroid use and hospitalisation over 6 years of follow-up. Gut 2016; 65: 408-14.

43 Turner D, Ricciuto A, Lewis A, et al. STRIDE-II: An Update on the Selecting Therapeutic Targets in Inflammatory Bowel Disease (STRIDE) Initiative of the International Organization for the Study of IBD (IOIBD): Determining Therapeutic Goals for Treat-to-Target strategies in IBD. Gastroenterology 2020; published online Dec 21. https://doi.org/10.1053/j.gastro.2020.12.031.

44 Danese S, Roda G, Peyrin-Biroulet L. Evolving therapeutic goals in ulcerative colitis: towards disease clearance. Nature Reviews Gastroenterology and Hepatology. 2020; 17: 1-2.

45 Christensen B, Hanauer SB, Erlich J, et al. Histologic Normalization Occurs in Ulcerative Colitis and Is Associated With Improved Clinical Outcomes. Clinical Gastroenterology and Hepatology 2017; 15: 1557-1564.e1.

46 Cushing KC, Tan W, Alpers DH, Deshpande V, Ananthakrishnan AN. Complete histologic normalisation is associated with reduced risk of relapse among patients with ulcerative colitis in complete endoscopic remission. Alimentary Pharmacology and Therapeutics 2020; 51: 347-55.

47 Magro F, Doherty G, Peyrin-Biroulet L, et al. ECCO Position Paper: Harmonization of the Approach to Ulcerative Colitis Histopathology. Journal of Crohn's \& colitis 2020; 14: 1503-11. 
48 Magro F, Estevinho MM, Dias CC, et al. Clinical, endoscopic and histological outcomes in induction of moderate-to-severe ulcerative colitis: a systematic review with meta-analysis. Journal of Crohn's and Colitis 2020; published online Sept 8. https://doi.org/10.1093/ecco-jcc/jjaa176.

49 Ma C, Guizzetti L, Panaccione R, et al. Systematic review with meta-analysis: endoscopic and histologic placebo rates in induction and maintenance trials of ulcerative colitis. Alimentary Pharmacology and Therapeutics. 2018; 47: 1578-96.

50 Magro F, Lopes J, Borralho P, et al. Comparison of different histological indexes in the assessment of UC activity and their accuracy regarding endoscopic outcomes and faecal calprotectin levels. Gut 2019; 68: 594-603.

51 Bossuyt P, Bisschops R, Vermeire S, de Hertogh G. Variability in the distribution of histological disease activity in the colon of patients with ulcerative colitis. Journal of Crohn's and Colitis 2020; published online Oct 14. https://doi.org/10.1093/ecco-jcc/jjaa206.

52 Singh S. Evolution of Clinical Trials in Inflammatory Bowel Diseases. Current Gastroenterology Reports. 2018; 20: 1-8.

53 Bhatt DL, Stone GW, Mahaffey KW, et al. Effect of Platelet Inhibition with Cangrelor during PCl on Ischemic Events. New England Journal of Medicine 2013; 368: 1303-13.

54 Léauté-Labrèze C, Hoeger P, Mazereeuw-Hautier J, et al. A Randomized, Controlled Trial of Oral Propranolol in Infantile Hemangioma. New England Journal of Medicine 2015; 372: 735-46.

55 Rugo HS, Olopade OI, DeMichele A, et al. Adaptive Randomization of Veliparib-Carboplatin Treatment in Breast Cancer. New England Journal of Medicine 2016; 375: 23-34.

56 Sato A, Shimura M, Gosho M. Practical characteristics of adaptive design in phase 2 and 3 clinical trials. Journal of Clinical Pharmacy and Therapeutics 2018; 43: 170-80.

57 Collignon $\mathrm{O}$, Koenig $\mathrm{F}$, Koch $\mathrm{A}$, et al. Adaptive designs in clinical trials: From scientific advice to marketing authorisation to the European Medicine Agency. Trials 2018; 19: 642.

58 Verstockt B, Noor NM, Marigorta UM, et al. Results of the Seventh Scientific Workshop of ECCO (III): Precision medicine in IBD: disease outcome and response to therapy. In press.

59 Ho GT, Porter RJ, Kalla R. Ulcerative colitis: Recent advances in the understanding of disease pathogenesis. F1000Research. 2020; 9: 294.

60 Seyed Tabib NS, Madgwick M, Sudhakar P, Verstockt B, Korcsmaros T, Vermeire S. Big data in IBD: Big progress for clinical practice. Gut. 2020; 69: 1520-32.

61 Fiocchi C, lliopoulos D. What's new in IBD therapy: An "omics network" approach. Pharmacological Research. 2020; 159: 104886.

62 Fiocchi C, Dragoni G, Iliopoulos D, Katsanos K, Hernandez Ramirez H SK. Results of the Seventh Scientific Workshop of the ECCO (I): Precision medicine in IBD - what, why and how. Journal of Crohn's \& Colitis 2020. In press. 


\section{Search strategy and selection criteria:}

We identified references for this review through searches of PubMed using the search terms "ulcerative colitis", "trial*", "remission", and "precision medicine" until December 2020. Only manuscripts published in English were reviewed. To identify recent results from ongoing clinical trials, we searched abstracts presented at the 2020 meetings of the European Crohn's and Colitis Organisation, United European Gastroenterology, and Digestive Diseases Week. The final reference list was generated based on relevance to the scope of this Review.

\section{Contributors}

DA: literature search, creating figures and drafting the manuscript. SV,BV and CF: literature search, supervision and critical revision of the manuscript. All authors agreed on the final manuscript.

\section{Declaration of interests}

B Verstockt reports financial support for research from Pfizer; lecture fees from Abbvie, Biogen, Chiesi, Falk, Ferring, Galapagos, Janssen, MSD, Pfizer, R-Biopharm, Takeda and Truvion; consultancy fees from Janssen, Guidepont and Sandoz.

S Vermeire reports financial support for research: MSD, AbbVie, Takeda, Pfizer, J\&J; Lecture fees from MSD, AbbVie, Takeda, Ferring, Centocor, Hospira, Pfizer, J\&J, Genentech/Roche; consultancy fees from MSD, AbbVie, Takeda, Ferring, Centocor, Hospira, Pfizer, J\&J, Genentech/Roche, Celgene, Mundipharma, Celltrion, SecondGenome, Prometheus, Shire, Prodigest, Gilead, Galapagos.

C Fiocchi received speaker fees from UCB, Sandoz, Janssen, Genentech, and consultancy fees from Athos Therapeutics.

D Alsoud declares no conflicts of interest

\section{Acknowledgments}

SV and BV received research grant from the Leona M. and Harry B. Helmsley Charitable Trust, and DA is funded by this grant. DA received also research grant from the IBD Patient's Association Flanders (CCV VZW) 
FIGURES

Therapy arm

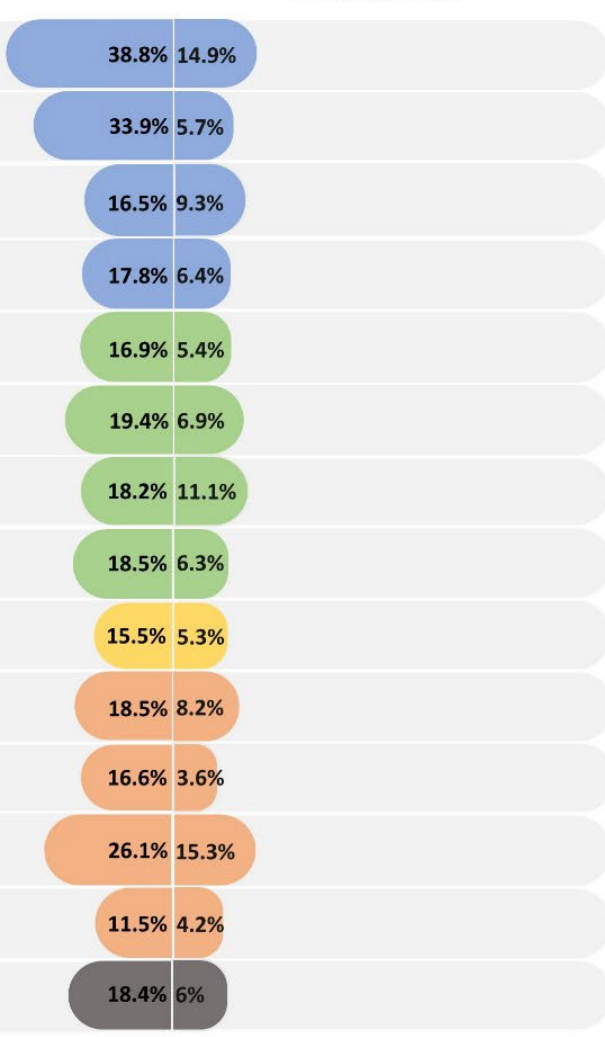

Trial - Drug

23.9\% ACT $1^{7}$ - Infliximab

28.2\% ACT $2^{7}-$ Infliximab

7.2\% ULTRA $2^{8}$ - Adalimumab

11.4\% PURSUIT-SC ${ }^{9}-$ Golimumab

11.5\% GEMINI ${ }^{10}$ - Vedolizumab

12.5\% HIBISCUS ${ }^{11}$ - Etrolizumab

7.1\% HIBISCUS II ${ }^{11}-$ Etrolizumab

12.2\% HICKORY ${ }^{12}$ - Etrolizumab

10.2\% UNIFI ${ }^{13}$ - Ustekinumab

10.3\% OCTAVE $1^{14}$ - Tofacitinib

13\% OCTAVE $2^{14}$ - Tofacitinib

10.8\% SELECTION $A^{15}$ - Filgotinib

7.2\% SELECTION B ${ }^{15}$ - Filgotinib

${ }_{12.4 \%}$ TRUE NORTH ${ }^{16}$ - Ozanimod

Figure 1. Remission rates in induction trials for ulcerative colitis 


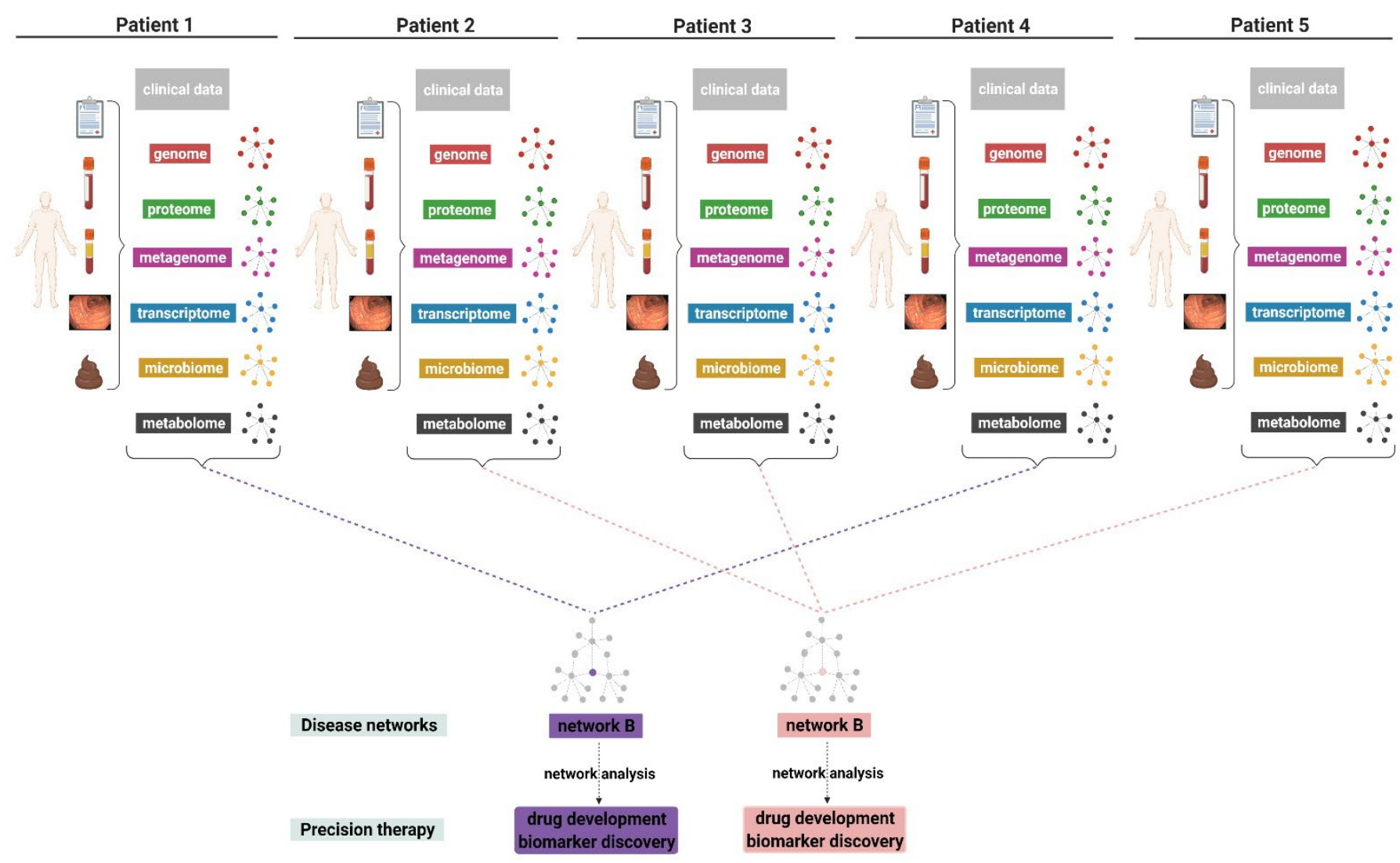

Figure 2. Omics approach to the identification of IBD networks

Figure 2.

An omics-based approach to the identification of IBD networks. Various omic data (genome, proteome, metagenome, transcriptome, microbiome, etc.) collected from the same patient generate individual networks, which are then integrated to generate different types of disease networks (network $A, B$, etc.) which represent distinct underlying molecular mechanisms. The identification of distinct disease networks and molecular subgroups allows the discovery of disease biomarkers associated with each type of network and, simultaneously, reveal the unique molecular controllers (hubs) of each IBD network that can then be therapeutically targeted to implement IBD precision therapy. 


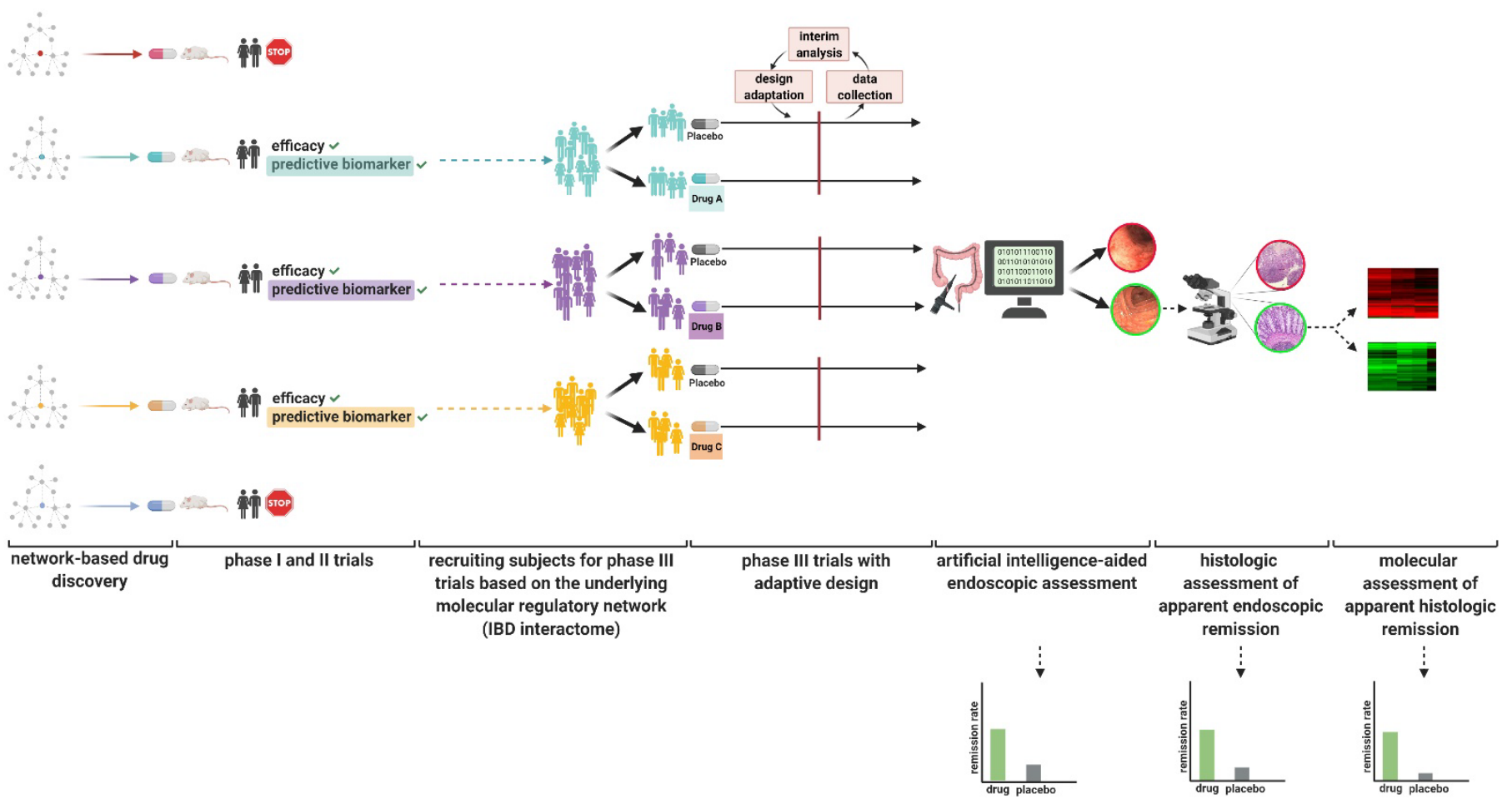

Figure 3. Proposed strategies to break the therapeutic ceiling in drug development in ulcerative colitis

Figure 3.

Proposed strategies to break the therapeutic ceiling in drug development in ulcerative colitis. Identification of distinct disease networks using omics integrating approach allows assigning individual IBD patients to distinct molecular subgroups, and for each subgroup a drug can be developed targeting the specific network hub. Applying this omics approach to biosamples collected during phase II trials reveals biomarkers predicting responsiveness or non-responsiveness, allowing to select ideal patients to participate in phase III trials administering network-based specific drug. Phase III trials with flexible adaptive designs can prove drugs' efficacy and identify optimal dosing regimens through efficient timeand cost-saving framework. New artificial intelligence-based endoscopic scoring systems can eliminate interobserver variability and demonstrate efficacy of investigational drugs over placebo. Additional histologic and molecular assessments further detect real therapeutic effects and lower false placebo response rates. 